the retroperitoneal tissues for a considerable distance allows the organ to be brought down when, before such a dissection, the proposition seemed hopeless. There is abundant evidence that the nutrition of the testis is dependant upon the integrity of the spermatic vessels, yet in some current text-books and works on operative surgery it is stated that in cases of difficulty these structures may be divided. Unless this teaching is entirely eradicated the prestige of orchidopexy is bound to suffer.

Multitudinous Methods of Orchidopexy.-There is hardly, if indeed there is, an operation in surgery where there are so many different methods of performance as that of orchidopexy. Advocates of a particular method are able to show that in their hands the operation is highly successful. When one critically examines the various methods, the main variation rests in the manner in which the organ is, as it were, splinted in the scrotum. The factor common to all showing a large percentage of successes is careful and thorough mobilization, particularly of the spermatic vessels. Without doubt this is the key-note of success and the method by which the organ is held in the scrotum is of secondary importance.

Conclusions :-

(I) The testis is an organ intolerant of pressure and for its proper development must be suspended in a loose sac-the scrotum.

(2) The gubernaculum prepares the path for the testis to descend, but the force which expels the organ from the abdomen must be sought for elsewhere.

(3) Natural descent after the second year is exceedingly rare.

(4) The age of election for performing orchidopexy is between the 7 th and IIth years.

(5) If the spermatic vessels are divided atrophy of the testis is inevitable.

(6) The common factor in the numerous methods of performing successful orchidopexy is thorough mobilization of the spermatic vessels without injuring them.

REFERENCES.

[1] Wangensteen, O. H. Arch. Surg., 1927, 663.

[2] Arey, L. B. "Developmental Anatomy," 1930.

[3] Goetsch, A. Amer. Journ. Surg., 1931, 63.

[4] JACOBSon. "Operations of Surgery." Sixth Edition. 1915.

[5] Browne, Denis. Lancet, 1933, i, 460.

\title{
THE TECHNIQUE OF ALKALI THERAPY IN CASES OF B.C.C. PYELITIS
}

\author{
BY A. ARNOLD OSMAN, D.s.C., F.R.C.P.,
}

Consulting Physician to St. Alfege Hospital, Greenwich, and Physician to Queen Mary's Hospital, London, $E$.

THOUGH alkalies have long been used in the treatment of acute and subacute infections of the urinary tract with Bacillus coli communis, and are almost universally regarded as the most potent agents for this purpose, experience shows that in a considerable proportion of cases the full benefit to be derived from their use is not attained owing to faulty technique in their administration. To give even fairly large doses of sodium bicarbonate and/or potassium citrate by mouth, and to rely on the urine turning red 
litmus paper blue as a result, may be sufficient in some cases, but in a large number it will only cause a temporary improvement, or none at all. The tendency then is to try other methods of treatment such as acid salts and hexamine, hexyl resorcin, mercurochrome, ketogenic diets, and so forth, when often all that is required is a careful adjustment of the dose of alkali given. In my experience, none of the substances and methods of treatment referred to above gives really satisfactory results in any large series of cases and over long periods of time, though in individual cases brilliant results are sometimes achieved with all of them. It is not only that the therapeutic results obtained are uncertain, but valuable time is lost in trying one form of treatment after another; meanwhile, an acute case is passing into the subacute or chronic stage. Large sums of money are also wasted annually by patients on expensive drugs (including vaccines under this heading), various forms of irrigation, both locally for the renal pelves and for the colon, and on expensive and unnecessary sojourns at spas and other places which cater for "renal" complaints. In the vast majority of cases, such therapeutic measures are as entirely unnecessary as they are usually ineffective. We have, in alkali therapy, properly carried out, a simple, cheap, and effective remedy for nearly all cases, and the attempt will now be made to set forth the details of a technique for the administration of alkalies which has rarely failed if undertaken conscientiously.

The essential factor in the treatment of these cases is the production of a satisfactory flow of urine, which by purely mechanical action flushes out the pelves and ureters, thereby preventing the formation of clots of inspissated pus and cellular debris, which are the chief causes of relapses, and finally of chronicity. Undoubtedly the production of a certain degree of alkalinity of the urine inhibits to some extent the continued growth of the B.C.C., but I am confident that this is a less important factor than is usually supposed, and far less important than the mechanical one due to an adequate diuresis. Recent observations, indeed, suggest that inhibition of growth is most satisfactorily accomplished by causing the urine to become unusually acid, as in the new ketogenic diet treatment of pyelitis. In actual practice, however, this latter method is unpleasant and not always successful. The essence of the alkaline method of treatment, then, is to induce and to maintain a satisfactory diuresis, and this is achieved by giving sufficient fluids and adequate doses of alkalies by mouth. If this be done, then marked improvement in signs and symptoms will almost invariably set in within forty-eight hours of the commencement of treatment. When satisfactory improvement does not occur, then either there is an obstruction in the urinary passages or the diagnosis has been mistaken. So true is this that (adequate) alkalinization may be used diagnostically and the presence of an obstruction confirmed by pyelography or other methods. This is particularly important in young children, as in two cases seen recently, in whom the diagnosis of obstruction was made and subsequently confirmed at operation, entirely on the lack of response to adequate alkali therapy.

\section{Regime.}

It will be convenient to consider the details of the method employed under separate headings.

(a) Rest.--Needless to say, all acute and subacute cases must be strictly confined to bed until the temperature has become quite normal, and all signs and symptoms such as local pain and tenderness, \&c., have cleared up. The temperature must be carefully taken and closely watched. At first, four-hourly readings will be required, but later a 
morning and evening reading will be sufficient. Rectal temperatures are not essential, but if mouth temperatures are taken it is important that the thermometer should be left in situ for not less than ten minutes. This applies to all thermometers whether "timed," " tested," "guaranteed" or not. If the temperature is worth taking at all it should be done with care. This is most important during the stage when the patient is first allowed up on a couch or chair. A slight rise of temperature requires either a readjustment of the dose of alkalies or sometimes a return to bed.

(b) Fluid Intake.-At least six pints of fluid as such must be consumed every twenty-four hours. Preferably eight pints should be aimed at but some patients find this difficult. The best fluids to give are water, barley-water, lemonade and orangeade, to any of which glucose may be added where severe anorexia prevents the taking of solid food. Skimmed milk with or without soda may also be given. It is the total quantity of fluid consumed that matters rather than its nature.

(c) Diet.-In all stages this can safely be left to the wishes of the patient. The acutely ill case with marked pyrexia, rigors, or vomiting, will not desire much and may be kept on fluids, with added glucose, and milk, which may be diluted if necessary. Some authorities are not in favour of giving milk at all, but provided the patient can tolerate it I have never seen any ill-effects from its use and generally give it as a routine in the early stages before solid food can be taken. Where large quantities of milk are being taken daily the amount should be included in the total fluid consumption for the twenty-four hours. Skimmed milk is often better tolerated than whole milk, and is especially useful in children in the acute stages. There is no special reason for limiting. the proteins as such in any stage, particularly as comparatively large doses of alkali are being given at the same time.

(d) Constipation.-There can be no doubt that B.C.C. infections of the urinary tract are closely associated with constipation, especially in women. Nevertheless, and contrary to accepted teaching, I believe purging in any stage to be unnecessary, and even unwise. Constipation can always be overcome by regular doses of liquid paraffin or petrolagar, and, if necessary, an occasional simple enema. In chronic cases the giving of paraffin may be supplemented by abdominal exercises and massage.

(e) Alkalies.-Experience has shown that the optimum degree of urinary alkalinity is that of a pH of 7.4 to $7^{\circ} 6$, that is to say, the urine must be made a little more alkaline than the blood normally is. Inasmuch as the early morning specimen (pre-breakfast) is usually the most acid of the day it is sufficient to ensure that this specimen is of the required degree of alkalinity, and if this be done then all subsequent specimens throughout the day will be even more alkaline, provided, of course, that the dose of alkali has not been altered. Should the early morning specimen become more acid than the optimum $\mathrm{pH}$ indicated, then a readjustment of dosage is called for. Testing the reaction $(\mathrm{pH})$, of the urine is an extremely easy matter occupying only a few seconds, and requiring. only the simplest apparatus, ${ }^{\prime}$ but it is important that the specimen (early morning) tested should be quite fresh, in fact it should be still warm, otherwise a false reading will be obtained. The method of testing consists essentially in sucking equal quantities (a few drops only) of urine and a standard dye (brom. thymol blue) into a capillary tube,

' A suitable and inexpensive apparatus is supplied by The British Drug Houses, Ltd., London, known as the "B.D.H. Capillator." 
mixing the liquids, and comparing the resulting colour with a series of standard coloured capillary tubes set in a cardboard frame. Testing the urine with litmus paper is not sufficiently accurate.

Dosage.-In acute cases with severe symptoms it is necessary to push the dosage rapidly. It has been found best to give orally a mixture of equal parts of sod. bic. and pot. cit. as large doses of sod. bic. Ialone tend to induce vomiting, whilst pot. cit. alone in appreciable amounts causes diarrhœa. By giving both salts in equal amounts these difficulties are avoided. I usually commence with the following mixture-

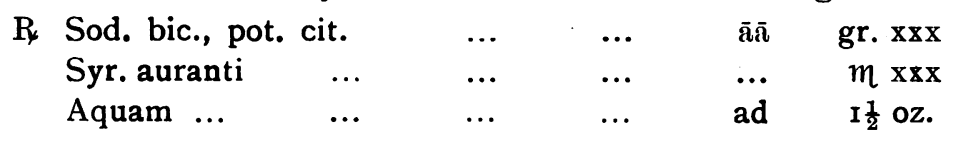

given either 2-, 3-, or 4-hourly according to the urgency of the case, and continue until the volume of urine passed is obviously increasing, and the reaction is definitely alkaline, i.e., $\mathrm{pH}=7.2$ or more. At this point the dose of alkali may generally be reduced to 3-, 4-, or 6-hourly so long as the urine remains at $\mathrm{pH} 7{ }^{\circ}$ or more. The dose which then maintains the early morning specimen of urine at $\mathrm{pH}_{7 \cdot 4}$ to $7^{\circ} 6$ is the optimum dose and must be continued as explained later. With rare exceptions, and where there is no mechanical obstruction to the flow of urine, a satisfactory diuresis (50-6o oz. per diem) and a sufficiently alkaline reaction is attained in about forty-eight hours. The urine is then practically clear to the naked eye, and the temperature and pain have disappeared. Thereafter, by trial, the dose may be reduced to that which will just maintain a $\mathrm{pH}$ of $7 \cdot 4$ to $7^{\circ} 6$ in the early morning specimens of urine. The medicine should be given strictly to time and in acute cases through the night as well. In subacute and chronic cases at least one dose should be given during the night. A slight increase of dosage may be called for when a more liberal diet is given, and especially when the patient first gets up and begins to walk. At such times it will usually be found that the urine has become slightly more acid and if this be not overcome a rise in temperature and recrudescence of symptoms will follow shortly. In early cases with intractable vomiting sod. bic. may be given per rectum as a three per cent. solution but this is rarely necesssary. Extra fluids may also be given per rectum in such cases. In a protracted case it is sometimes wise to change the flavouring agent in the mixture and aqua menth pip. with or without chloroform water is sometimes preferred. In most cases however there is little difficulty in getting patients to take the mixture as prescribed. When the patient has been up and about for some time and on a normal diet, cautious attempts may be made to reduce the dose of alkalies, but any return of pain, temperature, or turbidity of the urine, must be met by a resumption of the former dose. Although the urine usually becomes quite clear to the naked eye under this regime, microscopically some pus cells and B.C.C. may persist for months or years, and sometimes it is necessary for such cases to continue with alkalies for such periods. I have had cases on 400 and $500 \mathrm{gr}$. of alkali per diem for over two years in whom any attempt at reducing the dose was immediately followed by a return of symptoms.

The question now arises as to whether there is any danger in giving such comparatively large doses of alkalies without the aid of blood analyses. Fortunately there is none, or practically none, in uncomplicated cases, because the renal function is intact, and any excess of alkali is rapidly passed out in the urine. In a straightforward case then, a preliminary estimation of the blood alkali reserve is quite unnecessary. Such a 
procedure is only indicated where there is reason to believe that it may be increased above normal (alkalosis) before alkali therapy is begun. Alkalosis is likely to be met with in cases in whom there has been int ractable and copious vomiting for some days, or where for any reason there is marked and prolonged dyspnœa. Under such circumstances alkali therapy must be instituted slowly and with care, unless a preliminary estimation of the blood alkali reserve indicates that there is not already an alkalosis. When there is reason to believe that pyelitis is associated with chronic nephritis with nitrogen retention, similar precautions should be taken. Care is required also in cases with gross cardiac irregularities. I have never seen clinical alkalosis result from giving adequate doses of alkalies in uncomplicated cases.

\section{Pyelitis of Pregnancy.}

Acute and subacute B.C.C. pyelitis of pregnancy has certain features peculiar to this state. In pregnancy, at least in the later months, some element of mechanical obstruction is probably always present, and this of course tends to increase as pregnancy advances. In any stage of pregnancy, if the obstructive factor be marked then alkali therapy must inevitably fail. This is proved by the fact that cases not responding to adequate alkali treatment immediately improve on the introduction of ureteric catheters, or the termination of pregnancy. In early pregnancy, then, when obstruction, if present at all, is minimal in amount, thorough treatment with alkalies is to be recommended. In the later stages alkali therapy should also be given, and if not successful, and the disease appears to be threatening life, as occasionally happens, then pregnancy must be terminated. It is interesting to note that there is even less danger of alkalosis in pregnancy (unless complicated by chronic nephritis) than in normal persons, because? in this state the kidneys are unusually permeable to alkaline salts, so that any excess is readily passed out in the urine.

Experience in the treatment of many cases has shown that if the details of the method just described be conscientiously carried out, the results obtained will well repay both patient and physician. Lack of success is chiefly due to slovenly and haphazard dosage, or to a mistaken diagnosis.

\section{THE TREATMENT OF OBESITY.}

BY H. CECII, BULL, M.B., M.R.C.P.

THERE are two factors in the treatment of obesity-Psychology and Diet. Of these, Diet is simple. Psychology is very difficult, which explains why there is so much talk about weight reduction and so little accomplished.

"Everybody talking about Heaven ain't going there." The history of man is a record of individuals who have tried to control human wills-their own and those of others-with indifferent success. The teachings are beautiful enough and if men lived up to them heaven would be on earth and there would be no need to talk about it ; but there is an excess of wish and not enough will. Man is acutely conscious of this and in consequence suffers from psychological inferiority finding expression in a volume of talk and explanation-apology for not living up to the few simple rules of his belief. 